\title{
Scenedesmaceae (Chlorococcales, Chlorophyceae) de pesqueiros da Região Metropolitana de São Paulo, SP, Brasil: levantamento florístico ${ }^{1}$
}

\author{
Edna Ferreira Rosini ${ }^{2,3}$, Célia Leite Sant'Anna² e Andréa Tucci²
}

Recebido: 13.12.2012; aceito: 12.07.2013

\begin{abstract}
Scenedesmaceae (Chlorococcales, Chlorophyceae) from fishing ponds in São Paulo Metropolitan Region, São Paulo State, Brazil: a floristic survey). A survey on the Scenedesmaceae from 10 fishing ponds in São Paulo Metropolitan Region (RMSP) was performed. The samples were collected from the sub-surface, horizontally towed with a plankton net (20 $\mu \mathrm{m}$ mesh), in two periods: September-October 2001 (dry season) and February-March 2002 (rainy season), with a total of 20 samples. The samples were preserved in $4-5 \%$ formaldehyde solution, and studied under a photonic microscope. Twenty-six taxa of Scenedesmaceae were identified, distributed in 10 genera, 23 species, two varieties and one taxonomic form. Desmodesmus lefevrei (Deflandre) An, Friedl \& Hegewald, Dicloster acuatus Jao, Wei \& Hu and Scenedesmus baculiformis Chodat are cited for the first time for the State of São Paulo. Scenedesmus and Desmodesmus are the best represented genera, with nine and eight species, respectively.
\end{abstract}

Key words: Desmodesmus, Dicloster, Scenedesmus, taxonomy

RESUMO - (Scenedesmaceae (Chlorococcales, Chlorophyceae) de pesqueiros da Região Metropolitana de São Paulo, SP, Brasil: levantamento florístico). Foi realizado o levantamento das Scenedesmaceae em 10 pesqueiros da Região Metropolitana de São Paulo (RMSP). As amostras foram coletadas na sub-superfície por meio de arrasto horizontal com rede de plâncton $(20 \mu \mathrm{m})$, em dois períodos: setembro-outubro de 2001 (estiagem) e fevereiro-março de 2002 (chuvoso), totalizando 20 amostras. As amostras foram preservadas em formol a 4-5\% e estudadas ao microscópio fotônico. Foram identificados 26 táxons de Scenedesmaceae, distribuídos em 10 gêneros, 23 espécies, duas variedades e uma forma taxonômica. Desmodesmus lefevrei (Deflandre) An, Friedl \& Hegewald, Dicloster acuatus Jao, Wei \& Hu e Scenedesmus baculiformis Chodat são citadas pela primeira vez para o Estado de São Paulo. Scenedesmus e Desmodesmus foram os gêneros mais bem representados, com nove e oito espécies, respectivamente.

Palavras-chave: Desmodesmus, Dicloster, Scenedesmus, taxonomia

\section{Introdução}

Dentre as clorococales planctônicas, Scenedesmaceae é uma das famílias mais comumente encontradas nos sistemas aquáticos, seja pela riqueza de táxons existente ou pela distribuição cosmopolita de muitos dos seus representantes, como por exemplo, Scenedesmus e Desmodesmus.

Com base em análises morfológicas, a família foi classificada na ordem Chlorococcales (Chlorophyceae) (Komárek \& Fott 1983). No entanto, Krienitz et al. (2003) com base em estudos moleculares, incluíram a família Scenedesmaceae, exclusivamente autospórica, em Sphaeropleales, que compreende clorófitos unicelulares, filamentosos ou cenobiais que produzem células móveis biflageladas com corpos basais diretamente opostos. Estes mesmos autores incluíram espécies da família Coelastraceae em Scenedesmaceae, e Hegewald et al. (2010) incluíram a família Coelastraceae em Scenedesmaceae, com base em análises moleculares. Da mesma forma que membros de outras famílias foram adicionados em Scenedesmaceae, membros tradicionalmente pertencentes a essa família foram transferidos para

1. Parte da Dissertação do primeiro Autor, Programa de Pós-graduação em Biodiversidade Vegetal e Meio Ambiente, Instituto de Botânica

2. Instituto de Botânica, Núcleo de Pesquisa em Ficologia, Avenida Miguel Estéfano, 3687, 04301-012 São Paulo, SP, Brasil

3. Autor para correspondência: ednaferos@yahoo.com.br 
outros grupos, como Dicloster Jaó transferido para Trebouxiophyceae (Hegewald \& Hanagata 2000), Dydimocystis Korshikov para Oocystaceae (Hegewald 1988), Crucigeniella rectangularis (Nägeli) Komárek e Tetrachlorella alternans (G.M. Smith) Korsikov para Oocystaceae (Krienitz et al. 2003 e Hepperle et al. 2000, respectivamente).

Esses e outros estudos moleculares (ex. Buchheim et al. 2005, Krienitz \& Bock 2012, Leliaert et al. 2012) vêm apresentando uma nova imagem da filogenia das algas em geral, cujos resultados indicam o forte antagonismo com os critérios morfológicos aplicados na taxonomia e sistemática tradicionais, demonstrando casos em que semelhanças na morfologia nem sempre significam relações filogenéticas e casos de organismos morfologicamente distintos, porém filogeneticamente relacionados (Comas \& Sanches 2008).

Atualmente, 13 gêneros de Scenedesmaceae estão morfologica e filogeneticamente bem definidos. Vários outros gêneros dessa família, como todo o grupo de crucigenióideae, ainda estão sendo revisados (Krienitz \& Bock 2012).

As mudanças que estão ocorrendo na sistemática das Chlorophyceae, ainda não estão finalizadas e, portanto impossível de se apresentar um sistema de classificação plenamente atualizado (Comas \& Sanches 2008). Consequentemente, neste trabalho, para a distribuição dos gêneros dentro da família Scenedesmaceae utilizou-se o sistema tradicional de Komárek \& Fott (1983), mas foram consideradas as mudanças propostas por Hegewald (2000) para o gênero Scenedesmus e por Hepperle et al. (2000) para Tetrachlorella alternans.

O conhecimento sobre a comunidade fitoplanctônica em pesqueiros da Região Metropolitana de São Paulo está restrito aos seguintes trabalhos: Matsuzaki et al. (2004), Silva (2005), Sant'Anna et al. (2006), Gentil (2007) e Rosini et al. (2012). Dos 91 táxons registrados por Matsuzaki et al. (2004), 14 táxons pertencem à família Scenedesmaceae. Silva (2005) avaliou a dinâmica de populações de Microcystis (Cyanobacteria). Sant'Anna et al. (2006) e Gentil (2007) analisaram a estrutura da comunidade fitoplanctônica em 30 pesqueiros da Região Metropolitana de São Paulo. Sant'Anna et al. (2006) verificaram maior representatividade de clorofíceas e cianobactérias em termos de riqueza e abundância do fitoplâncton respectivamente. Gentil (2007) registrou 708 táxons em 30 pesqueiros, sendo
$13 \%$ de Scenedesmaceae, dos quais cinco táxons foram ilustrados. Rosini et al. (2012) identificaram 38 táxons distribuídos em oito famílias de Chlorococcales, exceto Scenedesmaceae.

Assim, o presente estudo será o primeiro trabalho especificamente taxonômico relativo à biodiversidade de Scenedesmaceae (Chlorococcales, Chlorophyceae) planctônicas em pesqueiros.

\section{Material e métodos}

A partir de 30 pesqueiros estudados por Gentil (2007), selecionaram-se aqueles com maior riqueza de espécies, totalizando 10 pesqueiros, localizados na Bacia Hidrográfica do Alto Tietê e identificados por códigos numéricos mantendo a sequência original proposta por Esteves \& Sant'Anna (2006), como segue: $8,9,10,11,14,16,18,25,27$ e 30 (figura 1). A bacia do Alto Tietê compreende a área drenada pelo rio Tietê a montante da Barragem de Pirapora até as suas nascentes em Salesópolis. Possui aproximadamente $37 \%$ de área urbanizada (Esteves \& Sant'Anna 2006).

As amostras foram coletadas na sub-superfície da coluna d'água com rede de plâncton, com abertura de malha de $20 \mu \mathrm{m}$ e foram preservadas em formol a $4-5 \%$. A profundidade média dos pesqueiros variou entre 1,0 e 1,5 m. As coletas foram realizadas em duas épocas: setembro-outubro de 2001 (final do período de estiagem) e fevereiro-março de 2002 (final do período chuvoso) $(n=20)$.

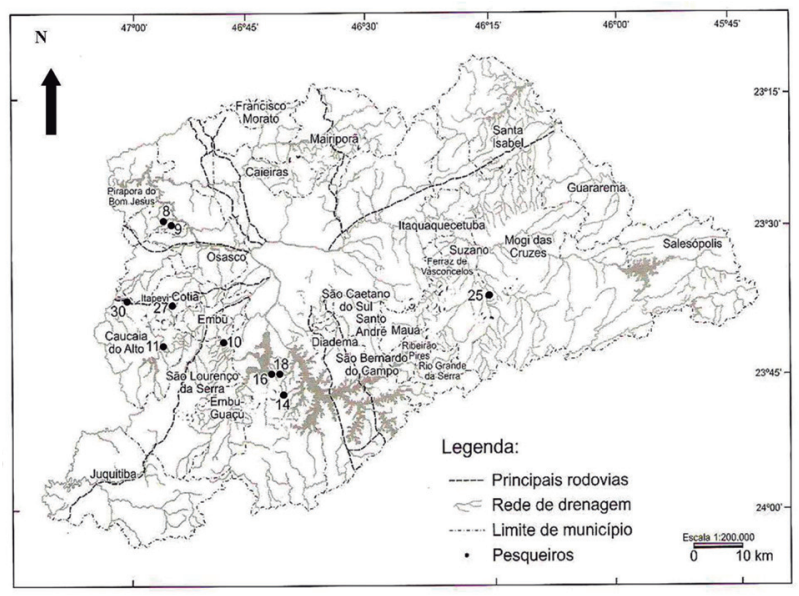

Figura 1. Localização dos pesqueiros estudados na Região Metropolitana de São Paulo, SP, Brasil (modificado de Presotto \& Cabianca 2006).

Figure 1. Location of the studied fishing ponds in São Paulo Metropolitan Region, São Paulo State, Brazil. (modified from Presotto \& Cabianca 2006). 
A identificação foi feita analisando-se as características morfológicas e métricas das populações de, no mínimo, 20 indivíduos de cada espécie. O exame das amostras foi realizado por meio de microscópio fotônico, Zeiss Axioplan 2, com câmara clara, retículo micrometrado e câmara fotográfica acoplados.

A distribuição geográfica foi feita com base em trabalhos especificamente taxonômicos realizados para o Estado de São Paulo: Leite (1974), Cardoso (1979), Sant'Anna (1984), Sant'Anna et al. (1988), Sant'Anna et al. (1989), Bicudo et al. (1992), Xavier (1996), Silva (1999), Peres \& Senna (2000), Ferragut et al. (2005), Tucci et al. (2006), Godinho (2009) e Rodrigues et al.(2010).

As amostras estão depositadas no acervo do Herbário Científico do Estado "Maria Eneyda P. Kauffmann Fidalgo" (SP), do Instituto de Botânica, São Paulo, SP.

\section{Resultados e discussão}

Foram identificados 26 táxons de Scenedesmaceae, distribuídos em 10 gêneros, 24 espécies, duas variedades e uma forma taxonômica. Entre os 26 táxons identificados, três são citados pela primeira vez para o Estado de São Paulo: Desmodesmus lefevrei, Dicloster acuatus e Scenedesmus baculiformis.

Tratamento taxonômico

\section{Scenedesmaceae}

\section{Crucigenia Morren 1830}

Crucigenia tetrapedia (Kirchner) W.West \& G.S.West, Transactions of the Royal Irish Academy: sér. B, 32: 62.1902.Basônimo:Staurogeniatetrapedia Kirchner, Jahresh. Ver. Vaterl. Naturk. Wurttemberg 36: 168, pl.II: fig. 1. 1880.

Figura 2a

Cenóbios planos, quadráticos, formados por 4 células dispostas cruciadamente; espaço central ausente; células triangulares, ca. 6,0 $\mu \mathrm{m}$ compr., ca. 3,0 $\mu \mathrm{m}$ diâm.; margens externas das células retas; cloroplasto único parietal com 1 pirenóide.

Material examinado: BRASIL. São PAULo: Pirapora do Bom Jesus, Pesqueiro 8, 17-IX-2001, K.E. Esteves s.n. (SP400164); Embu-Mirim, Pesqueiro 10, 19-IX-2001, K.E. Esteves s.n. (SP400159); idem, 26-II-2002, K.E. Esteves s.n. (SP400154); São Bernardo do Campo, Pesqueiro 14, 28-II-2002, K.E. Esteves s.n. (SP391352).

Distribuição geográfica no Estado de São Paulo: Municípios de São Paulo (Sant'Anna et al. 1989); Ribeirão Preto (Silva 1999); São Paulo (Ferragut et al. 2005, Tucci et al. 2006); Ortolândia, Pindamonhangaba, Pirassununga e São Paulo (Godinho 2009).

Crucigeniella Lemmermann 1900

Crucigeniella crucifera (Wolle) Komárek, Arch. Protistenk.116: 39. 1974. Basônimo: Staurogenia crucifera Wolle, Bull. Torrey Bot. Club. 6: 140. 1877.

Figuras 2b-c

Cenóbios planos, tetraédricos, formados por 4 células dispostas cruciadamente; espaço losangular no centro do cenóbio; células oblongas, levemente reniforme, 5,0-9,7 $\mu \mathrm{m}$ compr., 3,5-6,5 $\mu \mathrm{m}$ diâm.; parede celular espessada em um dos pólos; cloroplasto único parietal com um pirenóide.

Material examinado: BRASIL. São PAUlo: São Paulo, Embu-Mirim, Pesqueiro 10, 19-IX-2001, K.E. Esteves s.n. (SP400159); idem, 26-II-2002, K.E. Esteves s.n. (SP400154); Caucaia do Alto, Pesqueiro 11, 26-II-2002, K.E. Esteves s.n. (SP391350); Itapevi, Pesqueiro 30, 19-III-2002, K.E. Esteves s.n. (SP400158).

Distribuição geográfica no Estado de São Paulo: Municípios de São Paulo (Sant'Anna et al. 1989, Tucci et al. 2006, Rodrigues et al. 2010); Cosmorama e Itu (Godinho 2009).

Desmodesmus (R. Chodat) S.S. An, T. Friedl \& E. Hegewald 1999

An et al. (1999), com base na comparação da sequência do DNA ribossômico ITS-2, dividiram o gênero Scenedesmus Meyen em dois. As espécies que possuem espinhos nas células extremas e/ou intermediárias do cenóbio foram transferidas para Desmodesmus An, Friedl e Hegewald; e as espécies que não possuem espinhos nas células foram mantidas em Scenedesmus. Assim, Hegewald (2000) fez novas combinações para aquelas espécies bem caracterizadas que foram transferidas de Scenedesmus para Desmodesmus. Adotou-se no presente trabalho as novas combinações conforme Hegewald (2000). 

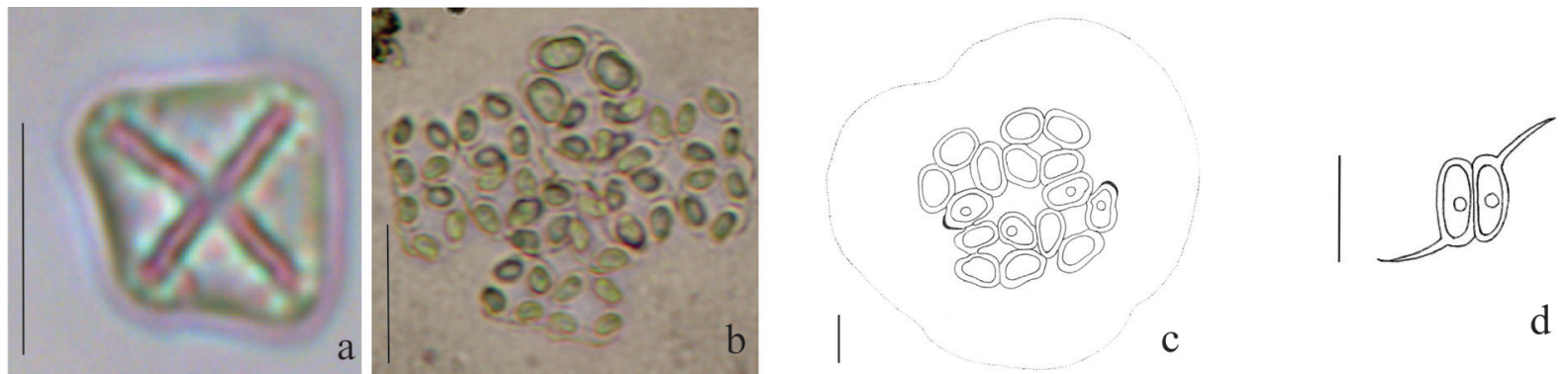

c

d
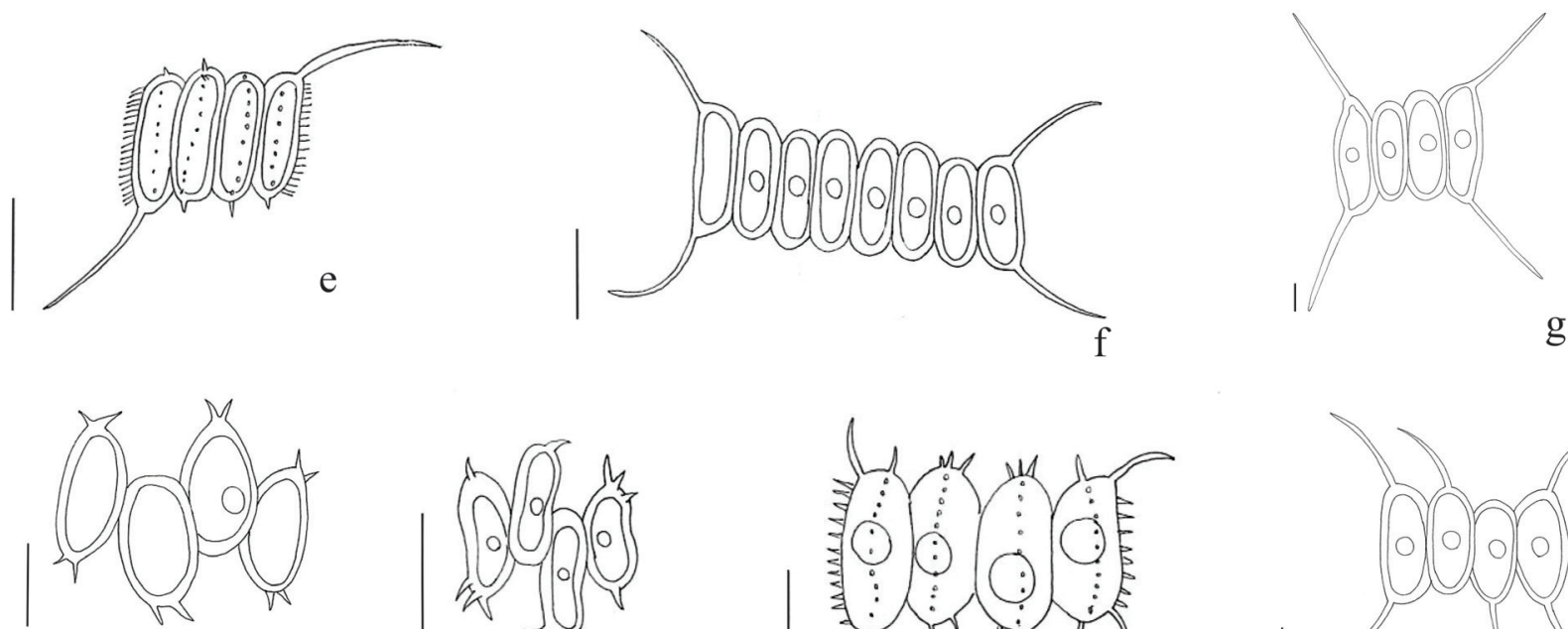

$\mathrm{h}$

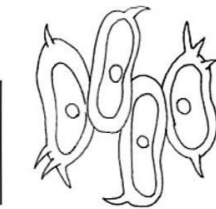

i
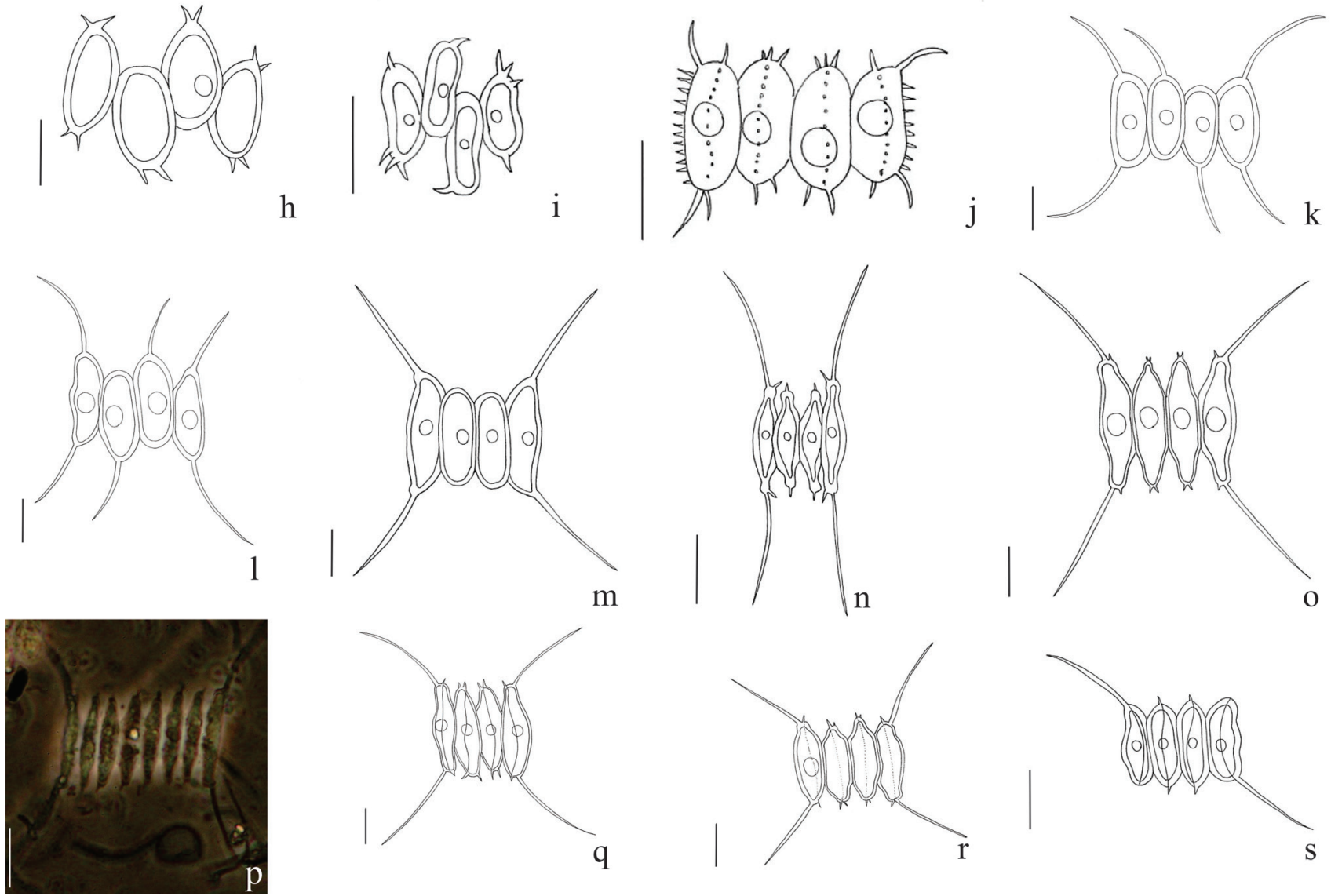

$\mathrm{p}$

Figura 2. Espécies de Scenedesmaceae de pesqueiros da Região Metropolitana de São Paulo, SP, Brasil. a. Crucigenia tetrapedia; b-c. Crucigeniella crucifera; d-e. Desmodesmus armatus var. bicaudatus; f-g. Desmodesmus communis; h-i. Desmodesmus denticulatus; j. Desmodesmus lefevrei; k-1. Desmodesmus maximus; m-p. Desmodesmus opoliensis; q-s. Desmodesmus opoliensis var. carinatus. Barra das escalas $=10 \mu \mathrm{m}$.

Figure 2. Scenedemaceae species found in the fishing ponds in São Paulo Metropolitan Region, São Paulo State, Brazil. a. Crucigenia tetrapedia. b-c. Crucigeniella crucifera. d-e. Desmodesmus armatus var. bicaudatus. f-g. Desmodesmus communis. h-i. Desmodesmus denticulatus. j. Desmodesmus lefevrei. k-1. Desmodesmus maximus. m-p. Desmodesmus opoliensis. q-s. Desmodesmus opoliensis var. carinatus. Scale bars $=10 \mu \mathrm{m}$. 
Chave para identificação das espécies de Desmodesmus encontradas nos pesqueiros estudados

1. Células externas apenas com 1 espinho em 1 dos pólos D. armatus var. bicaudatus

1. Células externas com 1 ou mais espinhos em cada um dos pólos

2. Células dispostas alternadamente D. denticulatus

2. Células dispostas linearmente

3. Células externas com pólos truncados

4. Células externas e internas com costelas longitudinais nas faces frontais ...... D. opoliensis var. carinatus

4. Células externas e internas sem costelas longitudinais D. opoliensis var. opoliensis

3. Células externas com pólos arredondados

5. Células externas naviculóides D. protuberans

5. Células externas oblongas, cilíndricas, ovóides ou elipsoides

6. Margem das células externas ornadas com até 2 espinhos acessórios

D. spinosus

6. Margem das células externas sem espinhos acessórios

7. Margem das células internas ornadas com uma fileira de espinhos

7. Margem da célula interna sem qualquer ornamentação

8. Células com até $17,6 \mu \mathrm{m}$ compr. D. lefevrei

8. Células acima de $20,0 \mu \mathrm{m}$ compr. D. communis D. maximus

Desmodesmus armatus var. bicaudatus (Guglielmetti) Hegewald, Algol. Stud. 96: 4. 2000. Basônimo: Scenedesmus acutiformis var. bicaudatus Guglielmetti,Nuova Notarisia, 21: 31. 1910.

Figuras 2d-e

Cenóbios planos, formados por 2-4 células dispostas linearmente; células oblongas, elipsoidais, 8,0-13,0 $\mu \mathrm{m}$ compr., 2,7-4,0 $\mu \mathrm{m}$ diâm.; células externas com 1 espinho em um dos pólos, dispostos em diagonal, 8,9-13,0 $\mu \mathrm{m}$ compr.; células internas sem espinhos polares; costelas longitudinais nas faces celulares terminadas em dentículos; cloroplasto parietal com 1 pirenóide.

Material examinado: BRASIL. São PAULO: São Paulo, Pirapora do Bom Jesus, Pesqueiro 8, 17-IX-2001, K.E. Esteves s.n. (SP400164); idem, Pesqueiro 9, 21-II-2002, K.E. Esteves s.n. (SP391349); São Bernardo do Campo, Pesqueiro 14, 24-IX-2001, K.E. Esteves s.n. (SP400161); idem, 28-II-2002, K.E. Esteves s.n. (SP391352); São Lourenço da Serra, Pesqueiro 16, 25-IX-2001, K.E. Esteves s.n. (SP400156); idem, 22-III-2002, K.E. Esteves s.n. (SP391354); idem, 18, 10-X-2001, K.E. Esteves s.n. (SP400160); Suzano, Pesqueiro 25, 8-X-2001, K.E. Esteves s.n. (SP400153); idem, 21-III-2002, K.E. Esteves s.n. (SP391357); Cotia, Pesqueiro 27, 10-X-2001, K.E. Esteves s.n. (SP400155); Itapevi, Pesqueiro 30, 19-III-2002, K.E. Esteves s.n. (SP400158).

Distribuição geográfica no Estado de São Paulo: Municípios de São Paulo (Leite 1974, Sant'Anna et al. 1989, Xavier 1996, Tucci et al. 2006 como
Scenedesmus bicaudatus (Hansgirg) Chodat e Rodrigues et al. 2010); São José dos Campos (Cardoso 1979, como S. bicaudatus (Hansgirg) Chodat); Americana, Atibaia, Barra Bonita, Guaratinguetá, Moji das Cruzes, Pindamonhangaba, Registro, Santo André, São Bernardo do Campo, São Paulo e Tupã (Sant'Anna 1984, como S. bicaudatus (Hansgirg) Chodat); Luiz Antônio (Peres \& Senna 2000, como S. bicaudatus (Hansgirg) Chodat); Arujá, Guaratinguetá, Rio Claro, Itaporanga, Paraguaçu Paulista, Pindamonhangaba e São Paulo (Godinho 2009).

As populações estudadas apresentaram indivíduos com características de Scenedesmus bicaudatus (figura 2d) e Scenedesmus semipulcher (figura 2e), os quais se diferenciam pela presença de costelas longitudinais nas faces celulares em Scenedesmus semipulcher. Embora sejam morfologicamente distintos, esses dois táxons foram sinonimizados por Hegewald (2000) como Desmodesmus armatus var. bicaudatus.

Desmodesmus communis (E. Hegewald) Hegewald, Algol. Stud. 96: 8. 2000. Basônimo: Scenedesmus communis E. Hegewald, Arch. Hidrobiol. Suppl. 51/ Algol. Stud. 19: 151, fig. 12 e 13. 1977.

Figuras 2f-g

Cenóbios planos, formadas por 4-8 células, dispostas linearmente; células oblongas 10,5-29,4 $\mu \mathrm{m}$ compr., 3,2-8,3 $\mu \mathrm{m}$ diâm.; células externas com 1 espinho em cada pólo, 10,5-25,0, 25,0 $\mu \mathrm{m}$ compr.; cloroplasto único parietal com 1 pirenóide. 
Material examinado: BRASIL. SÃo PaUlo: Pirapora do Bom Jesus, Pesqueiro 8, 17-IX-2001, K.E. Esteves s.n. (SP400164); idem, Pesqueiro 9, 21-II-2002, K.E. Esteves s.n. (SP391349); Embu-Mirim, Pesqueiro 10, 19-IX-2001, K.E. Esteves s.n. (SP400159); idem, 26-II-2002, K.E. Esteves s.n. (SP400154); São Lourenço da Serra, Pesqueiro 16, 25-IX-2001, K.E. Esteves s.n. (SP400156); idem, Pesqueiro 18, 10-X-2001, K.E. Esteves s.n. (SP400160); Suzano, Pesqueiro 25, 8-X-2001, K.E. Esteves s.n. (SP400153); idem, 21-III-2002, K.E. Esteves s.n. (SP391357); Cotia, Pesqueiro 27, 10-X-2001, K.E. Esteves s.n. (SP400155); idem, 19-III-2002, K.E. Esteves s.n. (SP391358); Itapevi, Pesqueiro 30, 19-III-2002, K.E. Esteves s.n. (SP400158).

Distribuição geográfica no Estado de São Paulo: Municípios de São Paulo (Leite 1974, Sant'Anna et al. 1989, Xavier 1996 e Tucci et al. 2006 como Scenedesmus quadricauda (Turpin) Brébisson, Ferragut et al. 2005 e Rodrigues et al. 2010); São José dos Campos (Cardoso 1979, como Scenedesmus quadricauda (Turpin) Brebisson); Arujá, Atibaia, Barra Bonita, Campos do Jordão, Cananéia, Guaratinguetá, Ibirá, Irapuã, Itirapina, Itu, Jaú, José Bonifácio, Juquiá, Miracatu, Moji das Cruzes, Pindamonhangaba, Rio Claro, Salesópolis, Santo André, São Bernardo do Campo, São Carlos, São Paulo, Sorocaba, Sumaré, Tupã e Ubatuba (Sant'Anna 1984, como Scenedesmus quadricauda (Turpin) Brébisson); Juquiá (Sant'Anna 1988, como Scenedesmus quadricauda (Turpin) Brébisson); Teodoro Sampaio (Bicudo et al. 1992, como Scenedesmus quadricauda (Turpin) Brebisson); Ribeirão Preto (Silva 1999, como Scenedesmus quadricauda (Turpin) Brebisson); Luiz Antônio (Peres \& Senna 2000, como Scenedesmus quadricauda (Turpin) Brebisson); Barra Bonita, Barretos, Guaratinguetá, Juquiá, Miracatu, Mirassol, Pindamonhangaba, Pirassununga, Rio Claro, Santo Anastácio, Santo André, São Paulo, Tremembé e Tupã (Godinho 2009).

Indivíduos com dimensões celulares semelhantes às de Scenedemus quadricauda var. maximus (27-36 × 9-11,5 $\mu \mathrm{m}$, Hegewald \& Silva 1988) foram identificados como a variedade típica devido a sequência contínua de medidas obtidas nas populações estudadas, o que dificultou estabelecer um limite entre as variedades maximus e quadricauda.
Desmodesmus denticulatus (Lagerheim) An, Friedl \& Hegewald, Algol. Stud. 96: 9. 2000. Basônimo: Scenedesmus denticulatus Lagerheim, Öfvers. K. Vetensk Akad. Forh. 39 (2): 61. pl. II, figs. 13-16. 1882.

Figuras 2h-i

Cenóbios planos, formados por 4 células, dispostas alternadamente em fileira única; células ovóides a elípticas ou alongadas cilíndricas, 8,0-16,2 $\mu \mathrm{m}$ compr., 4,0-8,1 $\mu \mathrm{m}$ diâm.; 1-3 espinhos polares; células internas com espinhos apenas em um dos pólos, 1,5-3,0 $\mu \mathrm{m}$ compr.; cloroplasto único, parietal, 1 pirenóide.

Material examinado: BRASIL. São PAULO: EmbuMirim, Pesqueiro 10, 19-IX-2001, K.E. Esteves s.n. (SP400159); Suzano, Pesqueiro 25, 8-X-2001, K.E. Esteves s.n. (SP400153); idem, 21-III-2002, K.E. Esteves s.n. (SP391357); Itapevi, Pesqueiro 30, 19-III-2002, K.E. Esteves S.n. (SP400158).

Distribuição geográfica no Estado de São Paulo: Municípios de São Paulo (Leite 1974, Sant'Anna et al. 1989, Xavier 1996 como Scenedesmus denticulatus Lagerheim e Rodrigues et al. 2010); São José dos Campos (Cardoso 1979, como S. denticulatus Lagerheim var. linearis Hansgirg); São Bernardo do Campo e São Paulo (Sant'Anna 1984, como S. denticulatus Lagerheim); Juquiá (Sant'Anna 1988, como S. denticulatus Lagerheim); Ribeirão Preto (Silva 1999, como S. denticulatus Lagerheim); Pilar do Sul e São Paulo (Godinho 2009).

Desmodesmus lefevrei (Deflandre) An, Friedl \& Hegewald, Algol. Stud. 96: 12. 2000. Basônimo: Scenedesmus lefevrei Deflandre, Bull. Soc. Bot. France 71: 673, pl. VI, fig. 12 e 16. 1924.

Figura $2 \mathrm{j}$

Cenóbios ligeiramente arqueados em vista lateral, formados por 4 células dispostas linearmente; células cilíndricas ou elipsóides, 11,3-15,3 $\mu \mathrm{m}$ compr., 4,8-7,0 $\mu$ m diâm.; células externas com 2 espinhos em cada pólo, um maior, 7,2-9,7 $\mu \mathrm{m}$ compr., outro menor, 4,0-5,0 $\mu \mathrm{m}$ compr.; células externas com 2 fileiras de espinhos curtos; células internas ornadas com 1 fileira de espinhos na região mediana terminando em pequenos espinhos polares; cloroplasto único parietal com 1 pirenóide.

Material examinado: BRASIL. São PAUlo: Pirapora do Bom Jesus, Pesqueiro 8, 17-IX-2001, K.E. 
Esteves s.n. (SP400164); Embu-Mirim, Pesqueiro 10, 19-IX-2001, K.E. Esteves s.n. (SP400159); Caucaia do Alto, Pesqueiro 11, 19-IX-2001, K.E. Esteves s.n. (SP400162); idem, 26-II-2002, K.E. Esteves s.n. (SP391350); São Lourenço da Serra, Pesqueiro 16, 25-IX-2001, K.E. Esteves s.n. (SP400156); Cotia, Pesqueiro 27, 10-X-2001, K.E. Esteves s.n. (SP400155); idem, 19-III-2002, K.E. Esteves s.n. (SP391358); Itapevi, Pesqueiro 30, 10-X-2001, K.E. Esteves s.n. (SP400157); idem, 19-III-2002, K.E. Esteves s.n. (SP400158).

Distribuição geográfica no Estado de São Paulo: Primeira citação.

Desmodesmus maximus (W.West \& G.S.West) Hegewald, Algol. Stud. 96: 13. 2000. Basônimo: Scenedesmus quadricauda var. maximus W.West \& G.S.West, Trans. Linn. Soc. London, Bot. 5: 83. 1895.

Figuras 2k-1

Cenóbios planos, formados por 4-8 células dispostas linear ou levemente alternadas; células cilíndricas, 20,0-31,5 $\mu \mathrm{m}$ compr., 6,1-11,3 $\mu \mathrm{m}$ diâm.; células externas com um espinho em cada pólo 20-26,7 $\mu \mathrm{m}$ compr., células internas com um espinho em um dos pólos, dispostos diagonalmente, ou espinhos ausentes; cloroplasto único parietal, 1 pirenóide.

Material examinado: BRASIL. São PAULo: Pirapora do Bom Jesus, Pesqueiro 9, 17-IX-2001, K.E. Esteves s.n. (SP400163); idem, 21-II-2002, K.E. Esteves s.n. (SP391349); Embu-Mirim, Pesqueiro 10, 19-IX-2001, K.E. Esteves s.n. (SP400159); idem, 26-II-2002, K.E. Esteves s.n. (SP400154); Suzano, Pesqueiro 25, 8-X-2001, K.E. Esteves s.n. (SP400153).

Distribuição geográfica no Estado de São Paulo: Municípios de Campos do Jordão, Guaratinguetá, Juquiá, São Carlos e São Paulo (Sant'Anna 1984, como Scenedesmus quadricauda var. maximus West \& West); São Paulo (Ferragut et al. 2005); Campos do Jordão, Ibirá, Juquiá e Tambaú (Godinho 2009).

Segundo Uherkovich (1966), S. quadricauda var. biornatus f. giganticus difere da var. biornatus pela maior dimensão celular: 30-40 × 8-12 $\mu \mathrm{m}$, na var. biornatus f. giganticus e 7-10 × 3-4,5 $\mu \mathrm{m}$, na var. biornatus. No material analisado, as populações apresentaram medidas intermediárias, 20,0-31,5 × 6,1-11,3 $\mu \mathrm{m}$, definindo assim, populações menores que as observadas por Uherkovich (1966). Outra diferença registrada em relação ao material ilustrado pelo autor é a disposição das células no cenóbio, alinhadas em Uherkovich (1966) e levemente alternadas nas populações deste estudo.

Desmodesmus opoliensis (P. Richter) Hegewald, Algol. Stud. 96: 14. 2000. Basônimo: Scenedesmus opoliensis P. Richter,Z.Angew. Mikrosk. Klin. Chem. 1: 7, figs. a-e. 1895.

Figuras $2 m-p$

Cenóbios planos, formados por 4-8 células dispostas linearmente; células externas naviculóides, pólos truncados com 1 espinho cada, ou 1 espinho e 1 dentículo, 13,7-26,0 $\mu \mathrm{m}$ compr., 3,2-8,0 $\mu \mathrm{m}$ diâm.; células internas oblongas, ou fusiformes truncadas menores que as externas $12,0-22,0 \mu \mathrm{m}$ compr., 3,2-8,6 $\mu \mathrm{m}$ diâm., sem espinhos, 1 ou 2 dentículos polares; cloroplasto parietal, 1 pirenóide.

Material examinado: BRASIL. São PAULO: Pirapora do Bom Jesus, Pesqueiro 9, 21-II-2002, K.E. Esteves s.n. (SP391349); Embu-Mirim, Pesqueiro 10, 19-IX-2001, K.E. Esteves s.n. (SP400159); idem, 26-II-2002, K.E. Esteves s.n. (SP400154); São Bernardo do Campo, Pesqueiro 14, 28-II-2002, K.E. Esteves s.n. (SP391352); São Lourenço da Serra, Pesqueiro 18, 19-III-2002, K.E. Esteves s.n. (SP391359); Suzano, Pesqueiro 25, 8-X-2001, K.E. Esteves s.n. (SP400153); idem, 21-III-2002, K.E. Esteves s.n. (SP391357); Itapevi, Pesqueiro 30, 10-X-2001, K.E. Esteves s.n. (SP400157); idem, 19-III-2002, K.E. Esteves s.n. (SP400158).

Distribuição geográfica no Estado de São Paulo: Municípios de São Paulo (Leite 1974, Sant'Anna et al. 1989, Ferragut et al. 2005, Tucci et al. 2006 como Scenedesmus opoliensis Richter e Rodrigues et al. 2010); São José dos Campos (Cardoso 1979, como S. opoliensis Richter); Jaú, Rio Claro, Santo André e São Bernardo do Campo (Sant'Anna 1984, como S. opoliensis Richter); Rio Claro, Santo André, São Bernardo do Campo e São Paulo (Godinho 2009).

As populações estudadas apresentaram grande variabilidade morfológica e a única característica que se mostrou constante foi o pólo truncado das células externas. Registraram-se indivíduos com células internas oblongas menores que as externas, e tamanho da região de contato igual ou superior a $2 / 3$ da extensão celular (figura $2 \mathrm{~m}$ ). Foram registrados ainda, indivíduos com células externas com 1 espinho 
e 1 dentículo polar; células internas fusiformes truncadas com 1 ou 2 dentículos polares e com a região de contato entre as células vizinhas, cerca de $1 / 3$ da extensão celular (figuras 2n-p). Outra característica marcante foi a disposição do espinho polar quase paralela ao eixo maior da célula (figura $2 n$ ). Esses últimos indivíduos assemelham-se a figura original de $S$. aristatus, ilustrado em Hegewald e Silva (1988), figura 125 p. 83, que segundo este autor, é sinônimo de S. opoliensis enquanto em Komárek \& Fott (1983) é sinônimo de $S$. protuberans var. minor. As populações estudadas foram identificadas como $S$. opoliensis de acordo com Uherkovich (1966), em função da semelhança com o polimorfismo ilustrado pelo autor.

Desmodesmus opoliensis var. carinatus (Lemmermann) Hegewald,Algol. Stud. 96: 15.2000.

Basônimo: Scenedesmus opoliensis var. carinatus Lemmermann, Forschungsber. Biol. Stat. Plon 7, 113, pl. fig.7. 1889.

Figuras 2q-s

Cenóbios planos, formados por 4 células dispostas linearmente; células externas oblongas, pólos truncados com 1 espinho e 1 dentículo cada, 15,0-20,2(-28,0) $\mu \mathrm{m}$ compr., 4,0-7,5(-10,0) $\mu \mathrm{m}$ diâm.; células internas elípticas, às vezes menores que as externas, 14,0-18(-25,0) $\mu \mathrm{m}$ compr., pólos arredondados truncados com 2 dentículos cada; células externas e internas com costelas longitudinais nas faces frontais; cloroplasto único parietal; 1 pirenóide.

Material examinado: BRASIL. São Paulo: Suzano, Pesqueiro 25, 8-X-2001, K.E. Esteves s.n. (SP400153); idem, 21-III-2002, K.E. Esteves s.n. (SP391357); Cotia, Pesqueiro 27, 10-X-2001, K.E. Esteves s.n. (SP400155); idem, 19-III-2002, K.E. Esteves s.n. (SP391358).

Distribuição geográfica no Estado de São Paulo: Municípios de São Paulo (Sant'Anna et al. 1989 e Tucci et al. 2006 como Scenedesmus carinatus (Lemmermann) Chodat); Arujá, Guaratinguetá, Juquiá, Miguelópolis e São Paulo (Godinho2009).

Em uma mesma amostra foram registrados indivíduos com características de $S$. opoliensis no que diz respeito às características das células externas do cenóbio, mas que se diferenciavam em dois morfotipos: grupo 1, apresentando indivíduos com células externas naviculóides, pólos truncados ou arredondados truncados com 1 espinho e 1 dentículo cada e células internas elipsóides com 1 ou dois dentículos polares (figuras $2 n-p$ ); grupo 2, apresentando as características acima descritas, somando a essas a presença de costelas nas faces celulares (figuras 2q-s). Hegewald (1979) incluiu na var. opoliensis indivíduos com ou sem costelas. Uherkovich (1995) ilustrou uma população sem costelas, com presença ou ausência de dentículos polares e a identificou como S. opoliensis; ilustrou ainda uma segunda população com costelas nas faces celulares e a identificou como S. carinatus; enquanto Comas (1996) apresentou indivíduos semelhantes a esta última população descrita por Uherkovich (1995) e os identificou como S. opoliensis var. carinatus. Percebe-se, portanto, que não havia unanimidade entre os autores quanto à nomenclatura dessas espécies e suas variedades. Assim, consideramos o grupo 1 como D. opoliensis var. opoliensis e o grupo 2 como $D$. opoliensis var. carinatus, conforme nova combinação proposta por Hegewald (2000).

Scenedesmus opoliensis var. carinatus difere da variedade típica, por apresentar costelas longitudinais nas faces frontais. É importante mencionar que poucos indivíduos nas populações estudadas apresentaram alterações no número e disposição dos espinhos, cujas células externas apresentaram somente um espinho, e este esteve diagonalmente oposto ao da outra célula externa (figura 2s). Essas alterações também foram observadas nas populações descritas por Nogueira (1991).

\section{Desmodesmus protuberans (Fritsch \& Rich)}

Hegewald, Algol. Stud. 96: 16. 2000. Basônimo:

Scenedesmus protuberans Fritsch \& Rich, Trans.

Roy. Soc. South Africa 18: 31, fig. 6. 1929.

Figuras 3a-c

Cenóbios planos, formados por 4 células dispostas linearmente; células externas naviculóides,

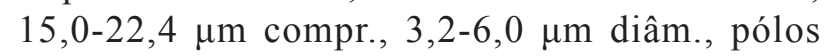
proeminentes arredondados com 1 espinho cada, 15-22 $\mu \mathrm{m}$ compr.; células internas oblongas ou elipsóides, 13,7-18,3 $\mu \mathrm{m}$ compr., 3,2-6,0 $\mu \mathrm{m}$ diâm., pólos arredondados ou levemente afilados; cloroplasto único parietal; 1 pirenóide.

Material examinado: BRASIL. São PAUlo: Pirapora do Bom Jesus, Pesqueiro 8, 17-IX-2001, K.E. Esteves s.n. (SP400164); idem, 21-II-2002, K.E. Esteves s.n. (SP391348); idem, Pesqueiro 9, 17-IX-2001, K.E. Esteves s.n. (SP400163); idem, 21-II-2002, K.E. Esteves s.n. (SP391349); Embu-Mirim, Pesqueiro 10, 19-IX-2001, K.E. Esteves s.n. (SP400159); idem, 
26-II-2002, K.E. Esteves s.n. (SP400154); Caucaia do Alto, Pesqueiro 11, 26-II-2002, K.E. Esteves s.n. (SP391350); São Lourenço da Serra, Pesqueiro 18, 19-III-2002, K.E. Esteves s.n. (SP391359); Suzano, Pesqueiro 25, 8-X-2001, K.E. Esteves s.n. (SP400153); idem, 21-III-2002, K.E. Esteves s.n. (SP391357); Cotia, Pesqueiro 27, 10-X-2001, K.E. Esteves s.n. (SP400155); idem, 19-III-2002, K.E. Esteves s.n. (SP391358); Itapevi, Pesqueiro 30, 10-X-2001, K.E. Esteves s.n. (SP400157); idem, 19-III-2002, K.E. Esteves s.n. (SP400158).

Distribuição geográfica no Estado de São Paulo: Municípios de Santo André, São Bernardo do Campo e São Paulo (Sant'Anna 1984, como S. protuberans Fritsch e Godinho 2009); São Paulo (Sant'Anna et al. 1989, Xavier 1996 e Tucci et al. 2006 como S. protuberans Fritsch); Teodoro Sampaio (Bicudo et al. 1992, como S. protuberans Fritsch); Ribeirão Preto (Silva 1999, como S. protuberans Fritsch) e Luiz Antônio (Peres \& Senna 2000, como S. protuberans Fritsch).

Desmodesmus spinosus (R. Chodat) Hegewald, Algol. Stud.96: 17.2000. Basônimo: Scenedesmus spinosus R. Chodat, Monogr. Alg. Cult. Purê: 74. pol. II: fig. 7. 1913.

Figuras 3d-h

Cenóbios planos, formados por 2-4 células dispostas linearmente; células externas oblongas, 8,1-9,0 $\mu \mathrm{m}$ compr., 2,4-3,2 $\mu \mathrm{m}$ diâm., face externa convexa com 1-2 espinhos, pólos arredondados com 1 espinho cada; células internas, 8,9-10,4 $\mu \mathrm{m}$ compr., 2,4-3,2 $\mu \mathrm{m}$ diâm., sem espinhos polares ou com 1 espinho em um dos pólos, dispostos diagonalmente, ou ainda, com 1 espinho em cada pólo; cloroplasto único parietal com 1 pirenóide.

Material examinado: BRASIL. S̃̃o PAulo: Suzano, Pesqueiro 25, 8-X-2001, K.E. Esteves s.n. (SP400153); Cotia, Pesqueiro 27, 10-X-2001, K.E. Esteves s.n. (SP400155); Itapevi, Pesqueiro 30, 10-X-2001, K.E. Esteves s.n. (SP400157); idem, 19-III-2002, K.E. Esteves s.n. (SP400158).

Distribuição geográfica no Estado de São Paulo: Municípios de São Paulo (Leite 1974 e Tucci et al. 2006 como S. abundans (Kirchner) Chodat, Ferragut et al. 2005); São José dos Campos (Cardoso 1979, como $S$. spinosus Chodat var. spinosus); Arujá, Atibaia, Rio Claro, Salesópolis, São Paulo, Sumaré e Tambaú (Sant'Anna 1984, como S. spinosus Chodat); Ribeirão
Preto (Silva 1999, como Scenedesmus spinosus Chodat); Arujá, Atibaia, Rio Claro, Salesópolis, Sumaré, Tambaú, Ribeirão Preto, São José dos Campos e São Paulo (Godinho 2009).

Segundo Godinho (2009), Desmodemus spinosus é facilmente confundido com Desmodesmus abundans (Kirchner) Hegewald, do qual difere pelo tamanho dos espinhos acessórios nas margens externas das células externas. Desmodesmus abundans possui 2 espinhos de tamanhos desiguais nas margens externas das células externas, podendo ocorrer isolados ou mais raramente em número de três, enquanto em D. spinosus os espinhos são praticamente do mesmo tamanho e em maior número.

Tendo em vista a grande dificuldade em separar essas duas espécies, alguns autores consideraram S. abundans sinônimo de $S$. spinosus, como Philipose (1967), Sant'Anna (1984), Nogueira (1991) e Uherkovich (1995).

Desmodesmus spinosus foi identificado de acordo com Uherkovich (1995). Semelhante ao ilustrado por este autor, a forma oblonga das células, a presença de quatro espinhos principais e a superfície lisa das faces celulares não variaram, enquanto que o número e a localização dos espinhos acessórios foram extremamente variáveis. Uma característica observada em alguns indivíduos dessa população que não foi mencionada em nenhum dos trabalhos analisados é a presença de células externas menores que as internas (figura 3e).

Dicloster Jao, Wei \& Hu 1976

Dicloster acuatus Jao, Wei \& Hu, Acta hydrobiol. sinica 6(1): 115, fig. 1-7. 1976.

Figuras $3 \mathrm{i}-\mathrm{j}$

Cenóbios formados por 4 células dispostas em 2 fileiras de células intercaladas, ou seja, a porção de união entre as células de uma fileira intercala-se com a outra; células lunadas, 34,0-60,0 $\mu \mathrm{m}$ de distância entre os ápices, 4,0-5,6 $\mu \mathrm{m}$ diâm., células unidas na porção convexa; cloroplasto único, parietal, incisão mediana conspícua, 2 pirenóides.

Material examinado: BRASIL. SÃo PAULO: Pirapora do Bom Jesus, Pesqueiro 8, 17-IX-2001, K.E. Esteves s.n. (SP400164); idem, 21-II-2002, K.E. Esteves s.n. (SP391348); idem, Pesqueiro 9, 17-IX-2001, K.E. Esteves s.n. (SP400163); idem, 21-II-2002, K.E. Esteves s.n. (SP391349); Embu-Mirim, Pesqueiro 10, 

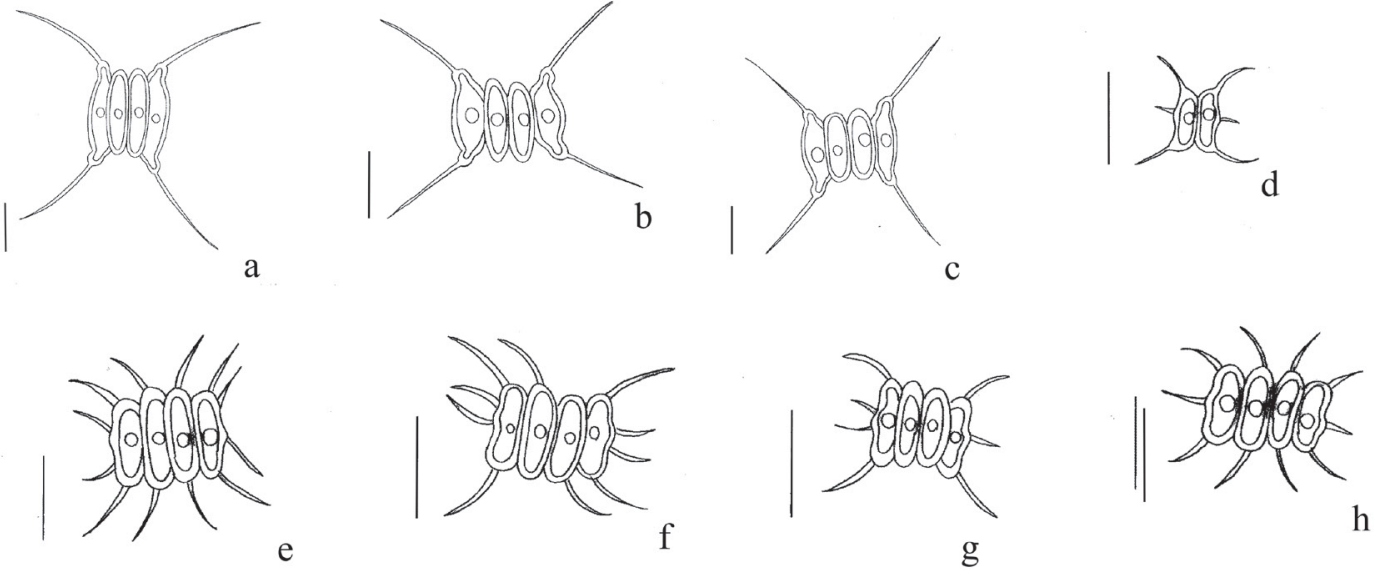

g

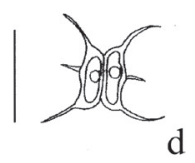

h
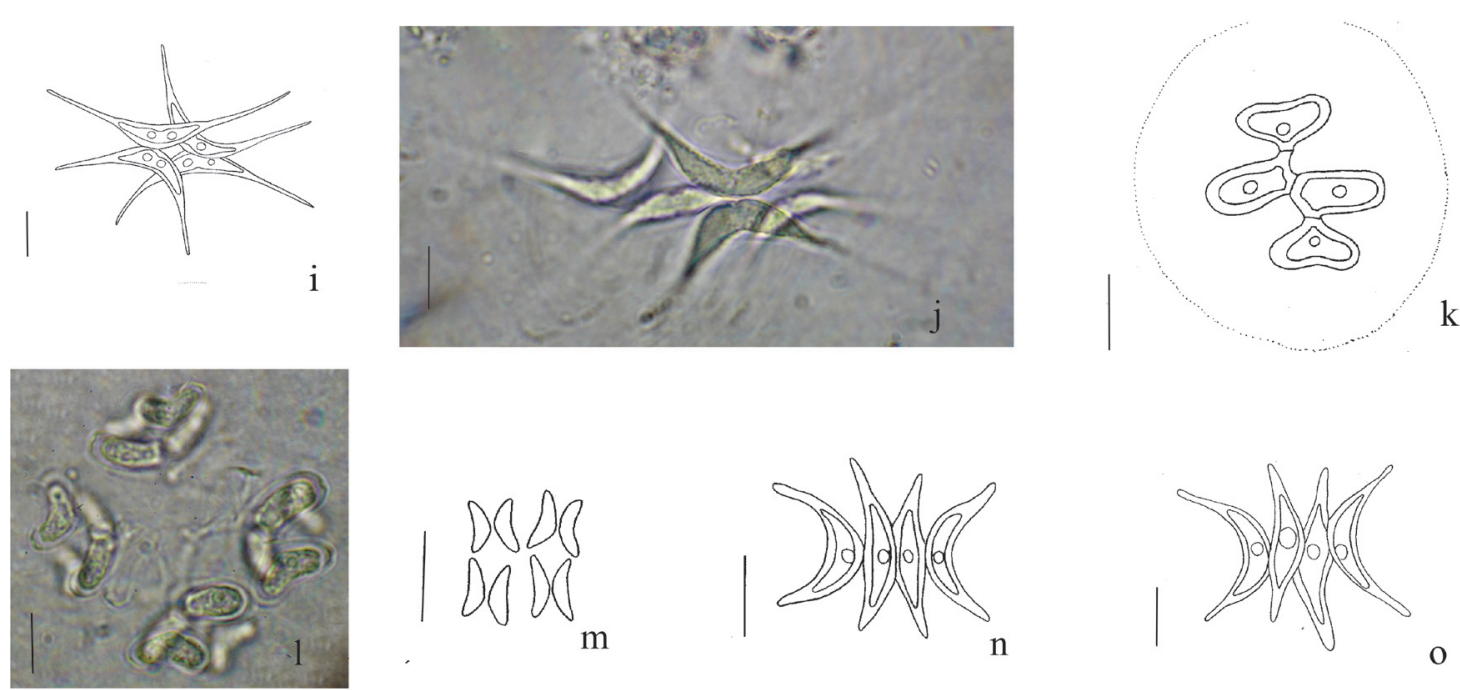

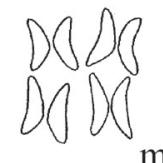

$\mathrm{m}$

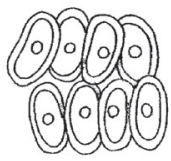

q
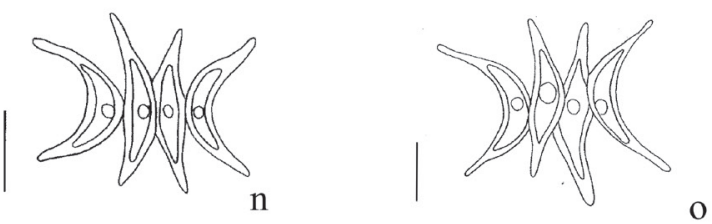

$\mathrm{O}$
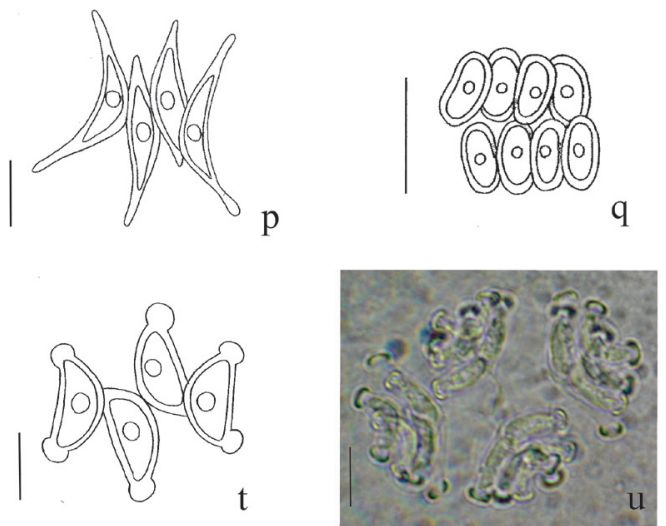
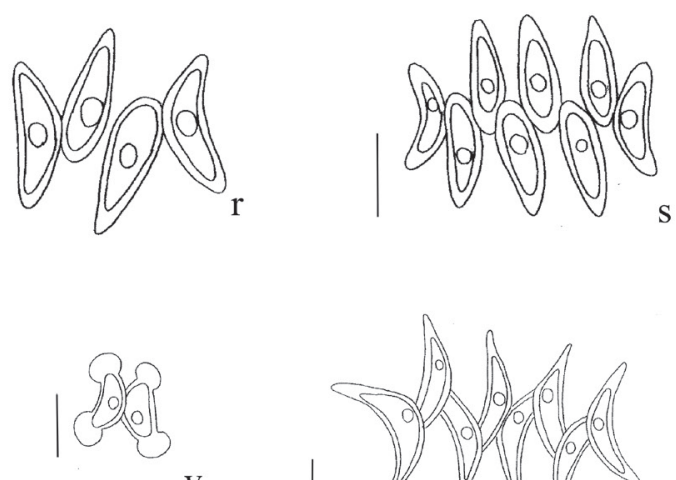

$\mathrm{V}$

W

Figura 3. Espécies de Scenedesmaceae de pesqueiros da Região Metropolitana de São Paulo, SP, Brasil. a-c. Desmodesmus protuberans. d-h. Desmodesmus spinosus. i-j. Dicloster acuatus. k-1. Dimorphococcus lunatus. m. Didymogenes palatina. n-p. Scenedesmus acuminatus. q. Scenedesmus arcuatus. r-s. Scenedesmus baculiformis. t-v. Scenedesmus indicus. u. Cenóbios em autoesporulação. w. Scenedesmus javanensis. Barra das escalas $=10 \mu \mathrm{m}$.

Figure 3. Scenedemaceae species found in the fishing ponds in São Paulo Metropolitan Region, São Paulo State, Brasil. a-c. Desmodesmus protuberans. d-h. D. spinosus. i-j. Dicloster acuatus. k-1. Dimorphococcus lunatus. m. Didymogenes palatina. n-p. Scenedesmus acuminatus. q. Scenedesmus arcuatus. r-s. Scenedesmus baculiformis. t-v. Scenedesmus indicus. u. Cenóbios em autoesporulação. w. Scenedesmus javanensis. Scale bars $=10 \mu \mathrm{m}$. 
26-II-2002, K.E. Esteves s.n. (SP400154); São Bernardo do Campo, Pesqueiro 14, 24-IX-2001, K.E. Esteves s.n. (SP400161); idem, 28-II-2002, K.E. Esteves s.n. (SP391352); São Lourenço da Serra, Pesqueiro 16, 22-III-2002, K.E. Esteves s.n. (SP391354); idem, Pesqueiro 18, 19-III-2002, K.E. Esteves s.n. (SP391359); Suzano, Pesqueiro 25, 21-III-2002, K.E. Esteves s.n. (SP391357); Cotia, Pesqueiro 27, 10-X-2001, K.E. Esteves s.n. (SP400155); idem, 19-III-2002, K.E. Esteves s.n. (SP391358); Itapevi, Pesqueiro 30, 10-X-2001, K.E. Esteves s.n. (SP400157); idem, 19-III-2002, K.E. Esteves s.n. (SP400158).

Distribuição geográfica no Estado de São Paulo: Primeira citação.

\section{Dimorphococcus Braun 1855}

Dimorphococcus lunatus A. Braun, Alg. unicell. p. 44. 1855.

Figuras 3k-1

Cenóbios esféricos, alongados, formados por 1 ou vários grupos de 4 células ligados por fios de mucilagem; células alternadas unidas por suas paredes, duas centrais oblongas e duas externas cordiformes, raramente reniformes, 11,3-13,7 $\mu \mathrm{m}$ compr., 4,0-6,5 $\mu \mathrm{m}$ diâm.; cloroplasto único parietal com 1 pirenóide.

Material examinado: BRASIL. São PAULo: Pirapora do Bom Jesus, Pesqueiro 9, 21-II-2002, K.E. Esteves s.n.
(SP391349); Embu-Mirim, Pesqueiro 10, 26-II-2002, K.E. Esteves S.n. (SP400154); São Lourenço da Serra, Pesqueiro 16, 25-IX-2001, K.E. Esteves s.n. (SP400156); idem, 22-III-2002, K.E. Esteves s.n. (SP391354); Itapevi, Pesqueiro 30, 19-III-2002, K.E. Esteves s.n. (SP400158).

Distribuição geográfica no Estado de São Paulo: Municípios de São Paulo (Leite 1974); Bauru, Cananéia, Guaratinguetá, Itu, Jaú, Moji das Cruzes, Porangaba, Rio Claro, São Bernardo do Campo, São Carlos, São Paulo, Sorocaba e Tambaú (Sant'Anna 1984); Luiz Antônio (Peres \& Senna 2000). Municípios de Guaratinguetá, Itu, e São Paulo (Godinho 2009).

Didymogenes Schmidle 1905

Didymogenes palatina Schmidle Hedwigia 45: 35, fig. 1-4. 1905.

Figura $3 \mathrm{~m}$

Cenóbios planos, formados por 8 células; células cilíndricas levemente arqueadas, unidas aos pares pelo lado convexo, dispostos paralelamente; pólos arredondados; ca. 7,3 $\mu \mathrm{m}$ compr., ca. 2,3 $\mu \mathrm{m}$ diâm.; cloroplasto único parietal; pirenóide não observado.

Material examinado: BRASIL. São PaUlo: Itapevi, Pesqueiro 30, 10-X-2001, K.E. Esteves s.n.(SP400157).

Distribuição geográfica no Estado de São Paulo: Município de São Paulo (Sant'Anna et al.1989, Tucci et al. 2006 e Godinho 2009).

Scenedesmus Meyen 1829

Chave para identificação das espécies de Scenedesmus encontradas nos pesqueiros estudados

1. Cenóbios formados por duas fileiras de células S. arcuatus

1. Cenóbios formados por uma fileira de células

2. Células dispostas linearmente

S. regularis

2. Células dispostas alternadamente

3. Células externas lunadas

4. Células internas ovais alongadas S. baculiformis

4. Células internas lunadas

5. Células unidas em suas regiões medianas S. acuminatus

5. Células com um dos pólos unindo-se a região mediana das células vizinhas

6. Distância entre os ápices celulares $20,2 \mu \mathrm{m}$ S. javanensis f. javanensis

6. Distância entre os ápices $28,3-56,0 \mu \mathrm{m}$ S. javanensis f. schroeteri

3. Células externas oblongas

7. Pólos celulares intumecidos S. indicus

7. Pólos celulares arredondados S. ovalternus 
Scenedesmus acuminatus (Lagerheim) R. Chodat, Beitr. Kryptogamenflora Schweiz, 1(3): 211. 1902. Basônimo: Selenastrum acuminatum Lagerheim, Ofvers. K. Vetensk Akad. Forh. 39(2): 71. 1882. Figuras 3n-p

Cenóbios formados por 4-8 células dispostas linearmente ou levemente alternadas, células unidas através das regiões medianas; células externas lunadas, células internas fusiformes levemente arcuadas, distância entre os ápices 12,1-29,9 $\mu \mathrm{m}$ compr., 2,4-4,8 $\mu \mathrm{m}$ diâm.; cloroplasto parietal com 1 pirenóide.

Material examinado: BRASIL. São PAULO: Pirapora do Bom Jesus, Pesqueiro 8, 17-IX-2001, K.E. Esteves s.n. (SP400164); idem, Pesqueiro 9, 21-II-2002, K.E. Esteves s.n. (SP391349); Embu-Mirim, Pesqueiro 10, 19-IX-2001, K.E. Esteves s.n. (SP400159); idem, 26-II-2002, K.E. Esteves s.n. (SP400154); São Bernardo do Campo, Pesqueiro 14, 28-II-2002, K.E. Esteves s.n. (SP391352); São Lourenço da Serra, Pesqueiro 16, 25-IX-2001, K.E. Esteves s.n. (SP400156); idem, 22-III-2002, K.E. Esteves s.n. (SP391354); idem, Pesqueiro 18, 19-III-2002, K.E. Esteves s.n. (SP391359); Suzano, Pesqueiro 25, 8-X-2001, K.E. Esteves s.n. (SP400153); idem, 21-III-2002, K.E. Esteves s.n. (SP391357); Cotia, Pesqueiro 27, 10-X-2001, K.E. Esteves s.n. (SP400155); Itapevi, Pesqueiro 30, 10-X-2001, K.E. Esteves s.n. (SP400157); idem, 19-III-2002, K.E. Esteves s.n. (SP400158).

Distribuição geográfica no Estado de São Paulo: Municípios de São Paulo (Leite 1974, Sant'Anna et al. 1989, Xavier 1996, Ferragut et al. 2005, Tucci et al. 2006 e Rodrigues et al. 2010). Americana, Atibaia, Bauru, Conchal, Guaratinguetá, Ibiúna, Irapuã, Itapeva, Jaú, Juquiá, Pontes Gestal, Registro, Santo André, São Bernardo do Campo, São Carlos, São Paulo, Sumaré e Tambaú (Sant'Anna 1984). Juquiá (Sant'Anna et al.1988); Teodoro Sampaio (Bicudo et al. 1992); Ribeirão Preto (Silva 1999, como Scenedesmus acuminatus (Lagerhemii) Chodat); Tambaú e Pirassununga (Godinho 2009).

As populações analisadas foram identificadas de acordo com Uherkovich (1995). Este autor analisou grandes populações, o que permitiu interpretar a variação morfológica encontrada e, além disso, apresentou várias ilustrações que refletem a variação morfológica encontrada nesta espécie.
As populações de $S$. acuminatus estudadas apresentaram indivíduos com células externas extremamente arcuadas e internas dispostas linearmente (figura 3n); outros com células externas menos curvadas e internas unindo-se da região próxima ao ápice da célula com a região mediana da célula subsequente, caracterizando disposição alternada levemente das células (figura $3 p$ ).

Scenedesmus arcuatus Lemmermann, ForschBer. Biol. Stat.Plön. 7: 112.1899. Basônimo: Scenedesmus bijugatus var. arcuatus Lemmermann, Bot. Centralbl. 76: 150. 1898.

Figura $3 \mathrm{q}$

Cenóbios curvos em vista apical, formados por 8 células dispostas alternadamente em duas fileiras; células oblongas ou levemente reniformes, 5,7-9,0 $\mu \mathrm{m}$ compr., 2,4-4,0 $\mu \mathrm{m}$ diâm.; cloroplasto único parietal; 1 pirenóide.

Material examinado: BRASIL. São Paulo: Pirapora do Bom Jesus, Pesqueiro 8, 17-IX-2001, K.E. Esteves s.n. (SP400164); idem, 21-II-2002, K.E. Esteves s.n. (SP391348); idem, Pesqueiro 9, 21-II-2002, K.E. Esteves s.n. (SP391349); São Lourenço da Serra, Pesqueiro 18, 19-III-2002, K.E. Esteves s.n. (SP391359); Cotia, Pesqueiro 27, 10-X-2001, K.E. Esteves s.n. (SP400155); idem, 19-III-2002, K.E. Esteves s.n. (SP391358); Itapevi, Pesqueiro 30, 10-X-2001, K.E. Esteves s.n. (SP400157); idem, 19-III-2002, K.E. Esteves s.n. (SP400158).

Distribuição geográfica no Estado de São Paulo: Municípios de São Paulo (Leite 1974, Sant'Anna et al. 1989, Xavier 1996 e Tucci et al. 2006); Itu e Santo André (Sant'Anna 1984).

Scenedesmus baculiformis Chodat, Z. Hydrol. 3: 158, fig.48. 1926.

Figuras 3r-s

Cenóbios formados por 4-8 células dispostas alternadamente; células com 8,3-16,2 $\mu \mathrm{m}$ compr., 4,0-4,8 بm diâm.; células externas lunadas, com a face externa côncava a quase reta, pólos arredondados; células internas ovais alongadas; cloroplasto único parietal; 1 pirenóide.

Material examinado: BRASIL. SÃo PAUlo: Pirapora do Bom Jesus, Pesqueiro 9, 21-II-2002, K.E. Esteves s.n. (SP391349); Itapevi, Pesqueiro 30, 19-III-2002, K.E. Esteves s.n. (SP400158). 
Distribuição geográfica no Estado de São Paulo: Primeira citação.

De acordo com a diagnose original, disponível em Hegewald \& Silva (1988), Scenedesmus baculiformis caracteriza-se por apresentar cenóbios formados por quatro células alternadas, assimétricas, baculiformis, suavemente curvadas. Comas (1996) considerou este táxon semelhante a $S$. obliquus (Turpin) var. obliquus e, portanto, questionou a identificação como um táxon independente. Komárek \& Fott (1983) comentaram que esta é uma espécie pouco conhecida e que pode ser uma metamorfose de $S$. bernardii. Santos (2008) registrou, como primeira citação para o Brasil, cenóbios formados por 8 células, apenas alguns formados por 4 células, com ampla variação morfológica e optou por mantê-los em Scenedesmus cf. baculiformis.

As populações analisadas concordam com o material descrito originalmente para $S$. baculiformis, apresentando cenóbios formados por 8 células.

Scenedesmus indicus Philipose, Indian Council of Agricultural Research 258. 1967.

Figuras 3t-v

Cenóbios formados por 4 células dispostas alternadamente; células com 12,1-16,0 $\mu \mathrm{m}$ compr., 4,0-5,6 스 diâm.; células externas arcuadas, pólos arredondados dilatados ou intumescidos, células internas oblongas com pólos intumescidos; cloroplasto único parietal, 1 pirenóide.

Material examinado: BRASIL. São PAULo: Pirapora do Bom Jesus, Pesqueiro 8, 21-II-2002, K.E. Esteves s.n. (SP391348); idem, Pesqueiro 9, 21-II-2002, K.E. Esteves s.n. (SP391349); Caucaia do Alto, Pesqueiro 11, 26-II-2002, K.E. Esteves s.n.(SP391350); Suzano, Pesqueiro 25, 21-III-2002, K.E. Esteves s.n. (SP391357); Itapevi, Pesqueiro 30, 19-III-2002, K.E. Esteves s.n. (SP400158).

Distribuição geográfica no Estado de São Paulo: Município de São Paulo (Tucci et al. 2006 e Godinho 2009).

Scenedesmus javanensis $\mathrm{R}$. Chodat. var. javanensis f. javanensis, Z. Hydrol. 3: 157, fig. 47. 1926. Figura 3w

Cenóbio formado por 8 células dispostas alternadamente; células externas lunadas, células internas assimétricas com um dos pólos ligando-se a região mediana da célula vizinha, exceto nas células centrais, que se unem através da região mediana; distância entre os ápices ca. 20,2 $\mu \mathrm{m}$ compr., ca. 4,8 $\mu \mathrm{m}$ diâm.; cloroplasto único parietal, 1 pirenóide.

Material examinado: BRASIL. São Paulo: São Paulo, Pesqueiro 25, 21-III-2002, K.E. Esteves s.n. (SP391357).

Distribuição geográfica no Estado de São Paulo: Municípios de São Paulo (Sant'Anna et al.1989, como Scenedesmus acuminatus (Lagerheim) Chodat var. bernardii (G.M. Smith) Dedusenko e Xavier 1996, como Scenedesmus javanensis R. Chodat); Ribeirão Preto (Silva 1999); Novo Horizonte e São Paulo (Godinho 2009).

Scenedesmus javanensis assemelha-se a $S$. bernardii pela disposição alternada das células. Entretanto, de acordo com descrição original em Hegewald \& Silva (1988), diferem entre si pelas células fusiformes com pólos agudos em $S$. bernardii e células fusiformes alongadas com os pólos gradualmente atenuados em $S$. javanensis. A taxonomia destas espécies esta baseada na forma e disposição das células nos cenóbios, e torna-se complicada devido a discordância entre os autores Smith (1916), Korsikov (1953), Uherkovich (1966), Komárek \& Fott (1983), Comas \& Komárek (1984), Sant'Anna (1984), Nogueira (1991), Comas (1996) e Rodrigues et al. (2010). Sendo assim, seguimos Chodat (1926), por considerarmos a forma da célula uma característica importante.

\section{Scenedesmus javanensis var. javanensis f. schroeteri}

(Hubernardii Chodatber-Pestallozi) Comas \& Komárek,ActaBot.Cubana 57:1-32.1988. Basônimo: Scenedesmus schroeteri Huber-Pestalozzi, Ber. Schweiz. Bot. Ges. 46: 153. fig. 5. 1936.

Figuras 4a-d

Cenóbios formados 4-8 células dispostas alternadamente; células externas levemente arcuadas, fusiformes retas ou com as margens externas levemente convexas; células internas assimétricas com um dos pólos ligando-se a região mediana das células vizinhas, exceto nas células centrais, que se unem através da região mediana; distância entre os ápices 28,3-56,0 $\mu \mathrm{m}$ compr., 4,0-7,2 $\mu \mathrm{m}$ diâm.; cloroplasto único parietal, 1 pirenóide.

Material examinado: BRASIL. SÃo PAULO: Pirapora do Bom Jesus, Pesqueiro 8, 17-IX-2001, K.E. Esteves s.n. (SP400164); idem, 21-II-2002, K.E. Esteves s.n. (SP391348); Embu-Mirim, Pesqueiro 10, 
19-IX-2001, K.E. Esteves s.n. (SP400159); idem, 26-II-2002, K.E. Esteves s.n. (SP400154); Caucaia do Alto, Pesqueiro 11, 26-II-2002, K.E. Esteves s.n. (SP391350); São Lourenço da Serra, Pesqueiro 18, 10-X-2001, K.E. Esteves s.n. (SP400160); Suzano, Pesqueiro 25, 8-X-2001, K.E. Esteves s.n. (SP400153); idem, 21-III-2002, K.E. Esteves s.n. (SP391357); Cotia, Pesqueiro 27, 10-X-2001, K.E. Esteves s.n. (SP400155); Itapevi, Pesqueiro 30, 10-X-2001, K.E. Esteves s.n. (SP400157); idem, 19-III-2002, K.E. Esteves s.n. (SP400158).

Distribuição geográfica no Estado de São Paulo: Município de São Paulo (Sant'Anna et al. 1989, como Scenedesmus acuminatus (Lagerheimii) Chodat $\mathrm{f}$. maximus Uherkovich e Godinho 2009).

Segundo Comas (1996), a forma schroeteri diferencia-se da forma típica por apresentar maior dimensão celular.
Scenedesmus ovalternus R. Chodat, Z. Hydrol. Hidrogr. Hidrobiol. 3 (3/4): 164, fig. 51. 1926.

Figura $4 \mathrm{e}$

Cenóbios formados por 8 células, dispostas alternadamente; células oblongas, 10,0-12,0 $\mu \mathrm{m}$ compr., 5,0-6,0 $\mu \mathrm{m}$ diâm.; cloroplasto único parietal, 1 pirenóide.

Material examinado: BRASIL. São Paulo: São Paulo, Pesqueiro 10, 19-IX-2001, K.E. Esteves s.n. (SP400159); idem, 26-II-2002, K.E. Esteves s.n. (SP400154).

Distribuição geográfica no Estado de São Paulo: Municípios de São Paulo (Leite 1974); Atibaia, Cananéia, Jaú, Ribeirão Branco, Salesópolis, São Bernardo do Campo, São Carlos, São Paulo e Ubatuba (Sant'Anna 1984) e São Paulo (Sant'Anna 1989, Ferragut et al. 2005 e Tucci et al. 2006).

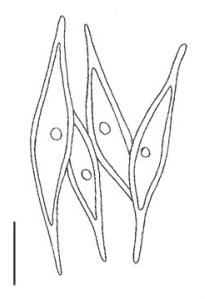

a

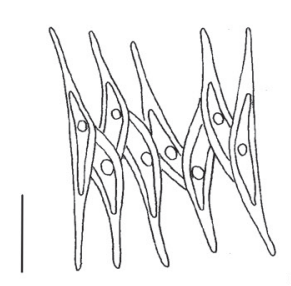

b

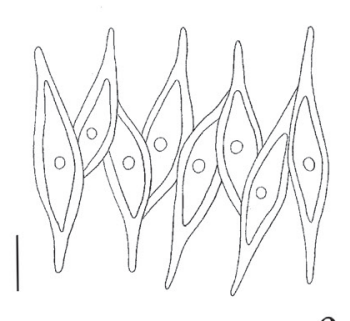

c

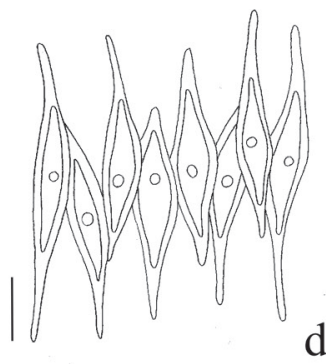

d
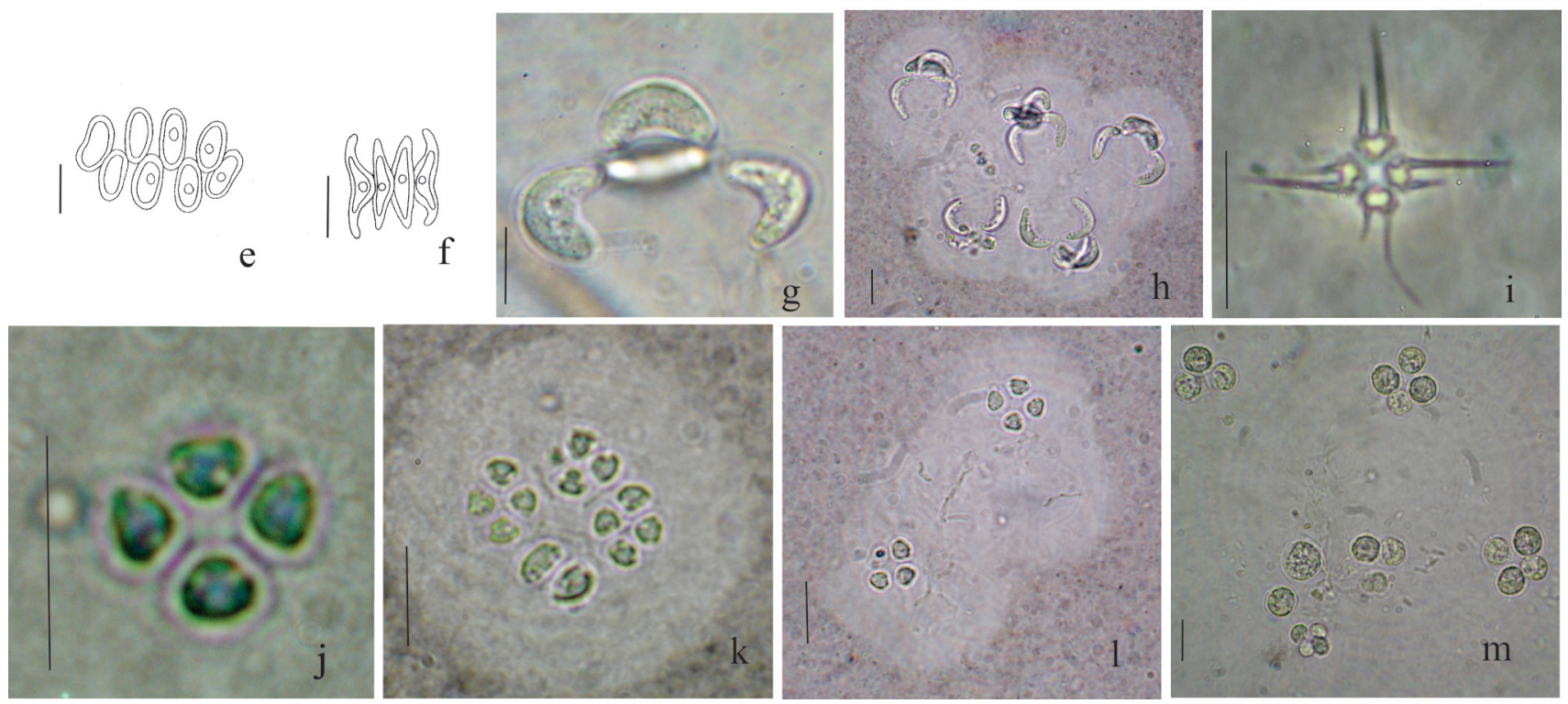

Figura 4. Espécies de Scenedesmaceae de pesqueiros da Região Metropolitana de São Paulo, SP, Brasil. a-d. Scenedesmus javanensis var. javanensis. f. schroeteri. e. Scenedesmus ovalternus. f. Scenedesmus regularis. g-h. Tetrallantos lagerheimii. i. Tetrastrum heteracanthum. j-1. Tetrastrum triangulare. $\mathrm{m}$. Westella botryoides. Barra das escalas $=10 \mu \mathrm{m}$.

Figure 4. Scenedemaceae species found in the fishing ponds in São Paulo Metropolitan Region, São Paulo State, Brazil. a-d. Scenedesmus javanensis var. javanensis. f. schroeteri. e. Scenedesmus ovalternus. f. Scenedesmus regularis. g-h. Tetrallantos lagerheimii. i. Tetrastrum heteracanthum. j-1. Tetrastrum triangulare. $\mathrm{m}$. Westella botryoides. Scale bars $=10 \mu \mathrm{m}$. 
Scenedesmus regularis Swirenko, Russk. Ark. Protistol. 3: 178. 1924.

Figura 4f

Cenóbios formados por 4 células fusiformes, dispostas linearmente, unidas pela região mediana das células, 13,0-17,0 $\mu \mathrm{m}$ compr., 3,0-4,0 $\mu \mathrm{m}$ diâm.; ápices das células externas curvados, voltando-se para o interior do cenóbio, faces externas das células marginais côncavas; células internas elipsóides; cloroplasto único parietal, 1 pirenóide.

Material examinado: BRASIL. São Paulo: Suzano, Pesqueiro 25, 8-X-2001, K.E. Esteves s.n. (SP400153).

Distribuição geográfica no Estado de São Paulo: Município de São Paulo (Tucci et al. 2006 e Godinho 2009).

\section{Tetrallantos Teiling 1916}

Tetrallantos lagerheimii Teiling, Svensk Botanisk Tidskrift 10: 62. 1916.

Figuras 4g-h

Cenóbios formados por 4 células, duas delas no mesmo plano, unidas pelos pólos, de modo que a face côncava de uma esteja voltada para a da outra, outras duas em outro plano, perpendicular ao anterior, cada uma tocando os pólos unidos do primeiro par; mucilagem hialina inconspícua; células lunadas, ápices arredondados, 8,0-15 $\mu \mathrm{m}$ compr. 4,0-7,0 $\mu \mathrm{m}$ diâm.; cloroplasto único, parietal; 1 pirenóide.

Material examinado: BRASIL. São PAULo: Pirapora do Bom Jesus, Pesqueiro 9, 21-II-2002, K.E. Esteves s.n. (SP391349); Caucaia do Alto, Pesqueiro 11, 26-II-2002, K.E. Esteves s.n. (SP391350); São Lourenço da Serra, Pesqueiro 18, 10-X-2001, K.E. Esteves s.n. (SP400160); idem, 19-III-2002, K.E. Esteves s.n. (SP391359); Suzano, Pesqueiro 25, 8-X-2001, K.E. Esteves S.n. (SP400153); idem, 21-III-2002, K.E. Esteves s.n. (SP391357); Cotia, Pesqueiro 27, 10-X-2001, K.E. Esteves s.n. (SP400155); Itapevi, Pesqueiro 30, 19-III-2002, K.E. Esteves s.n. (SP400158).

Distribuição geográfica no Estado de São Paulo: Municípios de Atibaia, Avaí, Guaratinguetá, Juquiá, Pindamonhangaba, Santo André, São Paulo, Sumaré e Ubatuba (Sant'Anna 1984); Juquiá (Sant'Anna et al. 1988); São Paulo (Sant'Anna et al. 1989, Xavier 1996, Ferragut et al. 2005 e Tucci et al. 2006); Ribeirão Preto (Silva 1999) Luiz Antônio (Peres \& Senna
2000); Capivari, Guaratinguetá, Jundiaí, São Paulo e Ubatuba (Godinho 2009).

\section{Tetrastrum Chodat 1895}

Tetrastrum heteracanthum (Nordstedt) Chodat, Alg. Vert. Suisse 209. 1902. Basônimo: Staurogenia heteracantha Nordstedt, Hedwigia 21: 106. 1882. Figura 4i

Cenóbios planos, formados por 4 células dispostas cruciadamente, deixando pequeno espaço central; células triangulares, ca. 4,0 $\mu \mathrm{m}$ diâm., margens em contato retas, margens externas côncavas com 2 espinhos retos de tamanhos desiguais, espinho maior 6,5-8,0 $\mu$ m compr., espinho menor, 2,5-4,0 $\mu \mathrm{m}$ compr.; cloroplasto único parietal; 1 pirenóide.

Material examinado: BRASIL. SÃo PAULO: Pirapora do Bom Jesus, Pesqueiro 8, 17-IX-2001, K.E. Esteves s.n.(SP400164); Embu-Mirim, Pesqueiro 10, 19-IX-2001, K.E. Esteves s.n. (SP400159); São Bernardo do Campo, 14, 28-II-2002, K.E. Esteves s.n. (SP391352); Itapevi, Pesqueiro 30, 19-III-2002, K.E. Esteves S.n. (SP400158).

Distribuição geográfica no Estado de São Paulo: Municípios de São Paulo (Leite 1974); Atibaia e Santo André (Sant'Anna 1984); São Paulo (Sant'Anna et al. 1989 e Tucci et al. 2006); Ribeirão Preto (Silva 1999); Guaratinguetá, Limeira e São Paulo (Godinho 2009).

Tetrastrum triangulare (Chodat) Komárek Archiv für Protistenkunde 116: 30. 1974. Basônimo: Staurogenia triangularis R. Chodat Mém. Herb. Boissier 1: 7-9. 1900.

Figuras $4 \mathrm{j}-1$

Cenóbios planos, quadráticos, formados por 4 células dispostas cruciadamente; espaço quadrático no centro do cenóbio; células trapezoidais 3,0-3,5 $\mu \mathrm{m}$ compr., 2,0-2,5 $\mu \mathrm{m}$ diâm.; margem externa convexa, margens laterais comprimidas pelo contacto com as células vizinhas; cloroplasto único parietal com 1 pirenóide.

Material examinado: BRASIL. São Paulo: EmbuMirim, Pesqueiro 10, 19-IX-2001, K.E. Esteves s.n. (SP400159); idem, 26-II-2002, K.E. Esteves s.n. (SP400154); São Bernardo do Campo, Pesqueiro 14, 28-II-2002, K.E. Esteves s.n. (SP391352); Suzano, Pesqueiro 25, 8-X-2001, K.E. Esteves s.n. (SP400153); Itapevi, Pesqueiro 30, 19-III-2002, K.E. Esteves s.n. (SP400158). 
Distribuição geográfica no Estado de São Paulo: Município de São Paulo (Sant'Anna et al.1989, Ferragut et al. 2005, Tucci et al. 2006); Guaratinguetá, Juquiá e Macedônia (Godinho 2009).

Westella De Wildermann 1897

\section{Westella botryoides (W. West) De-Wildeman} Bulletin de I'Herbier Boissier 5: 532.1897. Basônimo: Tetracoccus botryoides W. West, Journal of the Royal Microscopical Society 1892: 735. 1892.

Figura $4 \mathrm{~m}$

Cenóbios planos, quadrangulares, formados por 4 células dispostas cruciadamente; espaço intercelular retangular; sincenóbios frequentemente presentes, unidos por restos da parede da célula mãe; células esféricas, 6,4-10 $\mu \mathrm{m}$ diâm.; cloroplasto único poculiforme; 1 pirenóide.

Material examinado: BRASIL. SÃo Paulo: EmbuMirm, Pesqueiro 10, 19-IX-2001, K.E. Esteves s.n. (SP400159); São Bernardo do Campo, Pesqueiro 14, 24-IX-2001, K.E. Esteves s.n. (SP400161).

Distribuição geográfica no Estado de São Paulo: Municípios de São Paulo (Leite 1974); Atibaia e Santo André (Sant'Anna 1984) São Paulo (Sant'Anna et al. 1989 e Tucci et al. 2006); Ribeirão Preto (Silva 1999); Guaratinguetá, Limeira e São Paulo (Godinho 2009). Scenedesmus e Desmodesmus foram os gêneros melhores representados, com nove e oito espécies, respectivamente. Estes gêneros destacaram-se por apresentar grande polimorfismo, o que, por vezes, dificultou a identificação. Este fato ressalta a importância da avaliação de populações, bem como a ilustração dessas variações. Além disso, a frequente discordância entre os autores quanto à nomenclatura desses táxons, cujo polimorfismo acaba por confundir as características entre as espécies, constitui um problema para a identificação.

Desmodesmus spinosus (R. Chodat) Hegewald é um exemplo claro da problemática acima citada. Em Desmodesmus spinosus, a forma oblonga das células, a presença de quatro espinhos principais e a superfície lisa das faces celulares não variaram, enquanto o número e a localização dos espinhos acessórios foram extremamente variáveis; isso fez com que este táxon se assemelhasse a Desmodesmus abundans, o qual difere de $D$. spinosus, unicamente pelos 2 espinhos de tamanhos desiguais nas margens externas das células externas.

\section{Agradecimentos}

Ao CNPq pela concessão da bolsa de Mestrado para o primeiro Autor; à FAPESP processo $n^{\circ}$ 01/04081-8, pelo financiamento do projeto; a equipe de coleta do Instituto de Pesca da Secretaria de Abastecimento de São Paulo.

\section{Literatura citada}

An, S.S., Friedel, T. \& Hegewald E. 1999. Phylogenetic relationships of Scenedesmus and Scenedesmus-like coccoid green algae as inferred from ITS-2 rDNA sequence comparisons. Plantbiology 1: 418-428.

Bicudo, C.E.M., Bicudo, D.C., Castro, A.A.J. \& PicelliVicentim, M.M. 1992. Fitoplâncton do trecho a represar do Rio Paranapanema (Usina Hidrelétrica de Rosana), Estado de São Paulo, Brasil. Revista Brasileira de Biologia 52: 293-310.

Buchheim, M., Buchheim, J., Carlston, T., Braband, A., Hepperle, D., Krienitz, L. Hegewald, E. \& Wolf, M. 2005. Phylogeny of the Hydrodictyaceae (Chlorophyceae): Inferences From rDNA Data. Journal Phycology 41:1039-1054.

Cardoso, M.B. 1979. Ficoflórula da lagoa de estabilização de São José dos Campos, Estado de São Paulo, Brasil, exclusive Bacillariophyceae. Dissertação de Mestrado, Universidade de São Paulo, São Paulo.

Chodat, R. 1926. Scenedesmus: étude de génétique, de systematique experimentale et d'hydrobiologie. Zeitschrif für Hydrologie 3: 6-258.

Comas, A. \& Komárek, J. 1984. Taxonomy and nomenclature of several species of Scenedesmus (Chorellales). Algological Studies 35: 135-157.

Comas, A.G. 1996. Las Chlorococcales dulciacuícolas de Cuba. Gustav Fisher Verlag, Sttutgart.

Comas, A.G. \& Sánches, P. 2008. Algunas consideraciones y sugerencias sobre "La crisis em la taxonomia tradicional" con especial referencia a las algas verdes unicelulares (cocales). Boletín Sociedad Española de Ficologia Algas 39: 16-20.

Esteves, K.E. \& Sant'Anna, C.L. 2006. Pesqueiros sob uma visão integrada de meio ambiente, saúde pública e manejo. Rima, São Paulo.

Ferragut, C., Lopes, M.R.M., Bicudo, D.C., Bicudo, C.E.M. \& Vercellino, I.S. 2005. Ficoflórula perifítica e planctônica (exceto Bacillariophyceae) de um reservatório oligotrófico raso (Lago do IAG, São Paulo). Hoehnea 32: 137-184.

Gentil, R.C. 2007. Estrutura e dinâmica da comunidade fitoplanctônica de pesqueiros da Região Metropolitana de São Paulo, SP, em dois períodos: seca e chuva. Tese de Doutorado, Instituto de Botânica, São Paulo. 
Godinho, L.R. 2009. Família Scenedesmaceae no Estado de São Paulo: Levantamento florístico. Tese de Doutorado, Instituto de Botânica, São Paulo.

Hegewald, E. 1979. Vergleichende Beobachtungen an Herbarmaterial und Freilandmaterial von Scenedesmus. Arch. Hydrobiol. Suppl. 56: 264-486.

Hegewald, E. 1988. A new interpretation of the genus Didymocystis Korsikov (Chlorophyta, Chlorococcales). Algological Studies 48: 309-312.

Hegewald, E. 2000. New combinations in the genus Desmodesmus (Chorophyceae, Scenedesmaceae). Algological Studies 96: 1-18.

Hegewald, E. \& Silva, P. 1988. Annotated catalogue of Scenedesmus and nomenclaturally related genera including original descriptions and figures. J. Cramer, Stuttgart.

Hegewald, E. \& Haganata, N. 2000. Phylogenetic studies on Scenedesmaceae (Chlorophyta). Algological Studies 100: 29-49.

Hegewald, E., Wolf, M., Keller, A., Friedl, T. \& Krienitz, L. 2010. ITS2 sequence structure phylogeny in the Scenedesmaceae with special reference to Coelastrum (Chlorophyta, Chlorophyceae), including the new genera Comasiella and Pectinodesmus. Phycologia 49: 325-335.

Hepperle, D., Hegewald, E. \& Krienitz, L. 2000. Phylogenetic position of the Oocystaceae (Chlorophyta). Journal of Phycology 36: 590-595.

Komárek, J., \& Fott, B. 1983. Das Phytoplankton des Susswassers. Systematik und Biologie. Chlorophyceae (Grünalgen) Ordenung: Chlorococcales. E. Schweizerbart'sche verlagsbuchhandlung, Stuttgart.

Korshikov, A. 1953. Viznacnik prisnovodyych vodorostej Ukrajinskoji RSR, 5: Protococcineae, Kijev.

Krienitz, L. \& Bock, B. 2012. Present state of the systematics of planktonic coccoid green algae of inland waters. Hydrobiologia 698: 295-326.

Krienitz, L., Hegewald, E., Hepperle, D. \& Wolf, M. 2003. The systematics of coccoid grenn algae: $18 \mathrm{~S}$ rRNA gene sequence data versus morphology. Biologia 58: 437-446.

Leite, C.R. 1974. Contribuição ao conhecimento das Chlorococcales (Chlorophyceae) planctônicas do Parque Estadual das Fontes do Ipiranga, São Paulo, Brasil. Dissertação de Mestrado, Universidade de São Paulo, São Paulo.

Leliaert, F., Smith, D.R.,Moreau, H., Herron, M.D., Verbruggen, H., Deuwiche, C.F. \& Olivier, O.D. 2012. Phylogeny and molecular evolution of the Green Algae. Critical Reviews in Plant Sciences 31: 1-46.

Matsuzaki, M., Mucci, J.L.N. \& Rocha, A.A. 2004. Comunidade fitoplanctônica de um pesqueiro na cidade de São Paulo. Revista de Saúde Pública 38: 679-686.
Nogueira, I.S. 1991. Chlorococcales sensu lato (Chlorophyceae) do município do Rio de Janeiro e arredores, Brasil: inventário e considerações taxonômicas. Dissertação de Mestrado, Universidade Federal do Rio de Janeiro, Rio de Janeiro.

Peres, A.C. \& Senna, P.A.C. 2000. Chlorophyta da Lagoa do Diogo. In: J.E. Santos \& J.S.R. Pires. Estação Ecológica de Jataí. Rima, São Carlos, pp. 469-481.

Philipose, M.T. 1967. Chlorococcales. Indian Council of Agricultural Research Monographs, NewDelhi.

Presotto, A. \& Cabianca, M.A.A. 2006. Caracterização das Áreas de Localização de 30 Pesqueiros da Região Metropolitana de São Paulo. In: K.E. Esteves \& C.L. Sant'Anna (org.). Pesqueiros sob uma visão integrada de meio ambiente, saúde pública e manejo. Rima, São Paulo, pp.19-36.

Rodrigues, L.L., Sant'Anna, C.L. \& Tucci, A. 2010. Chlorophyceae das Represas Billings (Braço Taquacetuba) e Guarapiranga, SP, Brasil. Revista Brasileira de Botânica 33: 247-264.

Rosini, E.F., Sant'Anna, C.L. \& Tucci, A. 2012. Chlorococcales (exceto Scenedesmaceae) de pesqueiros da Região Metropolitana de São Paulo, SP, Brasil: levantamento florístico. Hoehnea 39: 11-38.

Sant'Anna, C.L. 1984. Chlorococcales (Chlorophyceae) do Estado de São Paulo, Brasil. Bibliotheca Phycologica 67: 1-348.

Sant'Anna, C.L., Xavier, M.B. \& Sormus, L. 1988. Estudo qualitativo do fitoplâncton da Represa de Serraria, Estado de São Paulo, Brasil. Revista Brasileira de Biologia 48: 83-102.

Sant'Ana, C.L., Azevedo, M.T.P. \& Sormus, L. 1989. Fitoplâncton do Lago das Garças, Parque Estadual das Fontes do Ipiranga, São Paulo, SP, Brasil: Estudo Taxonômico e Aspectos Ecológicos. Hoehnea 16: 89-131.

Sant'Anna, C.L., Gentil, R.C. \& Silva, D. 2006. Comunidade fitoplanctônica de pesqueiros da Região Metropolitana de São Paulo. In: K.E. Esteves \& C.L. Sant'Anna (org.). Pesqueiros sob uma visão integrada de meio ambiente saúde pública e manejo. Rima, São Paulo, pp. 49-62.

Santos, K.R.S. 2008. Biodiversidade de algas e cianobacterias de três lagoas ("salina", "salitrada" e "baia") do Pantanal da Nhecolândia, MS, Brasil. Dissertação de Mestrado, Instituto de Botânica, São Paulo.

Silva, D. 2005. Dinâmica de populações de Microcystis (Cyanobacteria) em pesqueiros da Região Metropolitana de São Paulo, SP, Brasil. Dissertação de Mestrado, Instituto de Botânica, São Paulo.

Silva, L.H.S. 1999. Fitoplâncton de um reservatório eutrófico (Lago Monte Alegre). Revista Brasileira de Biologia 59: 281-303. 
Smith, G.M. 1916. A monograph of the algal genus Scenedesmus based upon pure culture studies. Transactions of the Wisconsin Academy of Sciences, Arts and Letters 8: 422-530.

Tucci, A., Sant'Anna, C.L., Gentil, R.C. \& Azevedo, M.T.P. 2006. Fitoplâncton do Lago das Garças, São Paulo, Brasil: um reservatório urbano eutrófico. Hoehnea 33: 147-175.
Uherkovich, G. 1966. Die Scenedesmus Arten ungarns. Ungarischen. Akademiai Kiadó, Budapest.

Uherkovich, G. 1995. The green algae Scenedesmus (Chlorococcales Chlorophyceae) with special attention to taxa occurring in Hungary. Magyar Algologiai Fársaság, Budapest.

Xavier, M. B. 1996. Fitoplâncton do Rio Grande, Represa Billings, São Paulo, Brasil: Estudo Taxonômico (1985-1986). Iheringia 47: 103-122. 